\section{A New Improved Silicon Multi-Cathode Detector (SMCD) for Microanalysis and $X$-Ray Mapping Applications}

Shaul Barkan*, Valeri D. Saveliev*, Jan S. Iwanczyk*, Liangyuan Feng ${ }^{\star}$, Carolyn R. Tull ${ }^{\star}$, Bradley E. Patt*, Dale E. Newbury ${ }^{\star *}$, John A. Small ${ }^{\star *}$, and Nestor J. Zaluzec ${ }^{\star * *}$ ${ }^{*}$ Radiant Detector Technologies, ${ }^{* * N a t i o n a l ~ I n s t i t u t e ~ o f ~ S t a n d a r d s ~ a n d ~}$ Technology, ${ }^{* *}$ Electron Microscopy Center, Argonne National Lab

A silicon multi-cathode detector (SMCD) has been developed for microanalysis and $\mathrm{x}$-ray mapping applications $[1,2]$. The SMCD has a large active area $\left(\sim 0.5 \mathrm{~cm}^{2}\right)$, excellent energy resolution, and high count rate capability. The detector utilizes novel structures that have produced very low dark current, high electric field, uniform charge collection, low noise and high sensitivity to low energy $\mathrm{x}$ rays $[3,4]$.

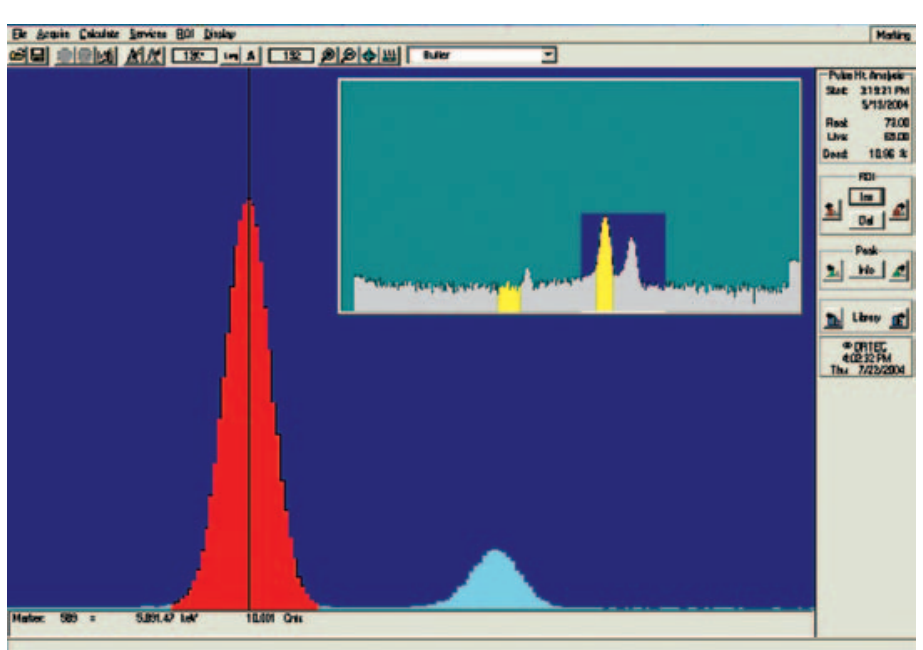
Fig radioisotope source, as well as by fluorescing materials with an $\mathrm{x}$-ray generator. Figure 1 shows a ${ }^{55} \mathrm{Fe}$ spectrum with an energy resolution of $125 \mathrm{eV}$ FWHM at $5.9 \mathrm{keV}$ collected at $12 \mu$ seaking time. This energy resolution has been repeatably measured on many different detectors. To evaluate the high count rate $\mathrm{x}$-ray performance, which is very important for fast $\mathrm{x}$-ray mapping, a Cu sample was fluoresced using a Rh-anode $\mathrm{x}$-ray tube. Figure 2 shows the energy resolution as a function of count rate for the $\mathrm{Cu} \mathrm{K}_{\alpha}$ line, while Figure 3 shows the $\mathrm{Cu} \mathrm{K}$ photopeak position as a function of count rate. As can be seen from these measurements, the photopeak position and energy resolution are virtually independent of count rate, which is significantly important for high speed mapping application.

A custom-designed spectrometer package was built with a long probe to fit a JEOL 840 scanning electron microscope (SEM) at the National Institute of Standards and Technology (NIST). This spectrometer package is shown in Figure 4. In order to verify the fast $\mathrm{x}$-ray mapping capability of the SMCD, a Raney nickel alloy sample (an aluminum-nickel alloy that is used in hydrocarbon processing) was analyzed on the JEOL 840 SEM. The SAMx (France) spectrum imaging software was used with the conditions of $128 \times 128$ pixels and $10 \mathrm{~ms}$ dwell time (with $1.3 \mathrm{~ms}$ overhead), which resulted in a total collection time of only $185 \mathrm{~s}$. The electron beam energy and
The detector's spectral response was evaluated using a ${ }^{55} \mathrm{Fe}$

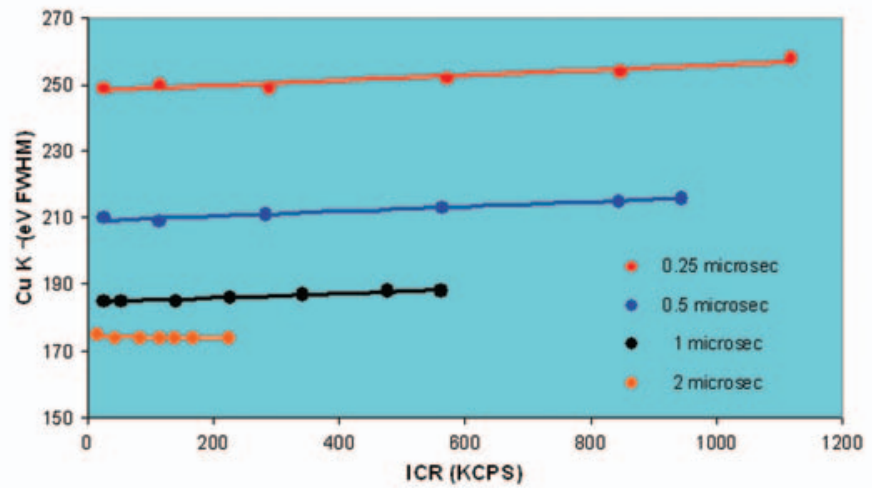

Fig. 2. Cu $K_{\alpha}$ energy resolution (FWHM) as a function of incoming count rate (ICR), from 0.25 - $2 \mu$ s peaking time.

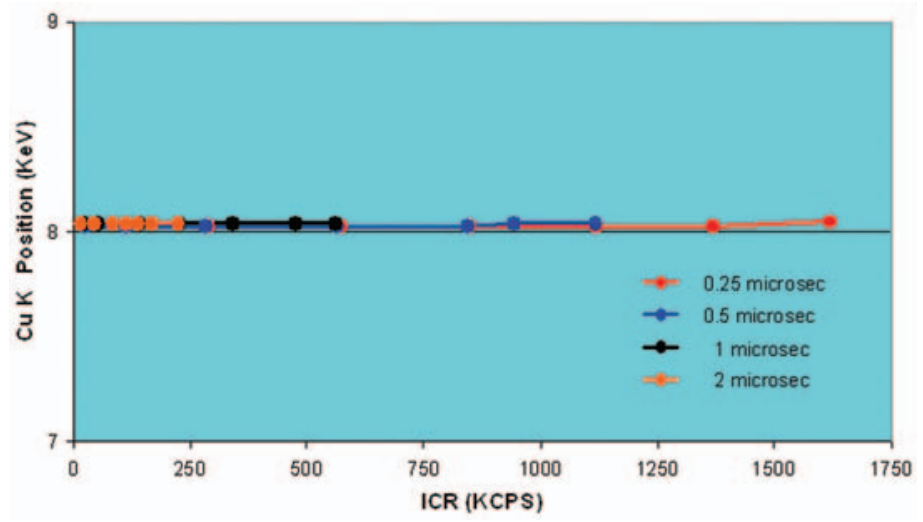

Fig. 3. $\mathrm{Cu} K_{\alpha}$ peak position as a function of count rate.

current was $20 \mathrm{keV}$ and $10 \mathrm{nA}$, respectively, and the pulse processor peaking time was $0.5 \mu$ s. The results of the mapping measurements are shown in Figure 5. As seen in Figure 5, the structure in the map in the starting (as-cast) material consists of dendrites of nickel-rich composition, surrounded by an aluminum-rich phase (about $99.5 \% \mathrm{Al}-0.5 \% \mathrm{Ni}$ ). To activate the Raney nickel for use, this Al-rich phase is etched out in an acid treatment, leaving the dendrites behind as a highly complex, three-dimensional structure with a high surface-to-volume ratio, ideal for catalysis.

A conventional state-of-the art energy dispersive spectrometer (such as a $\mathrm{Si}(\mathrm{Li})$ or HPGe detector) operating at the same energy

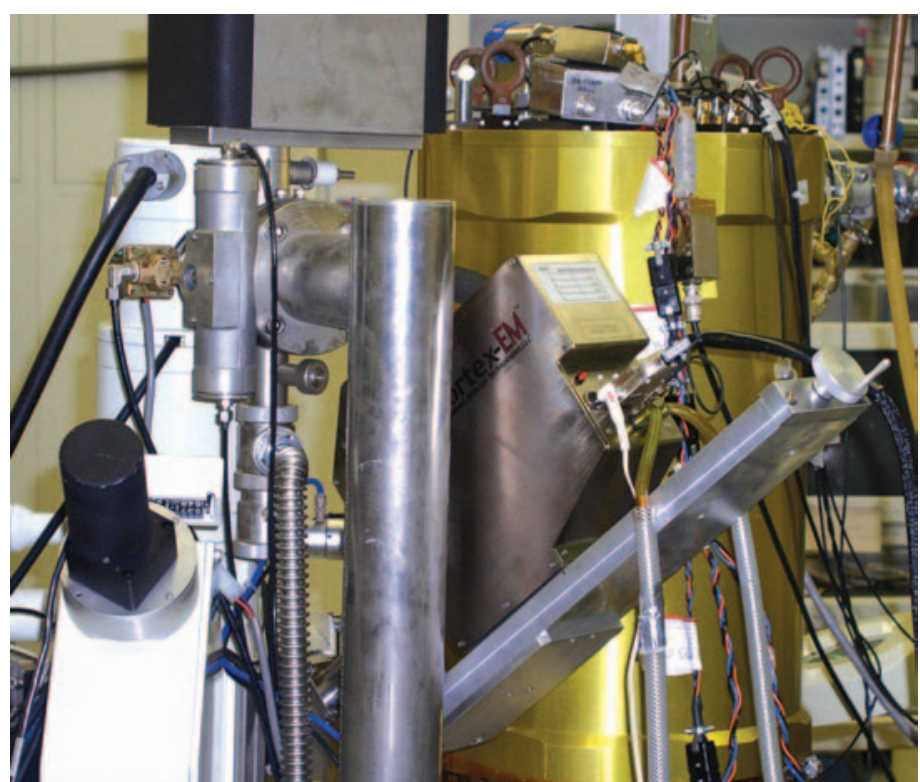

Fig. 4. SMCD mounted on a JEOL 840 SEM at NIST 


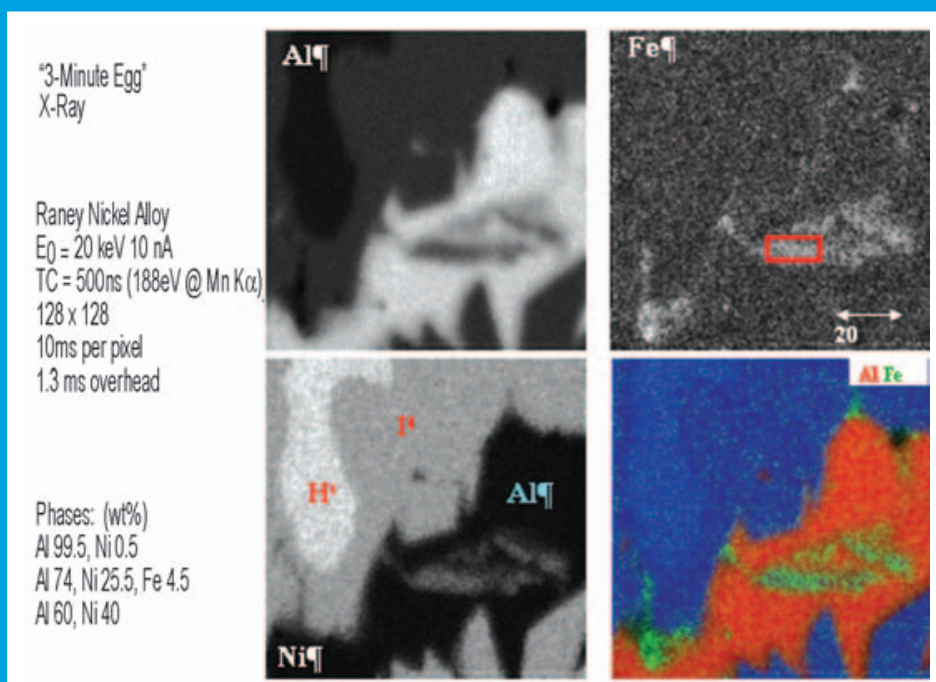

Fig. 5. X-ray maps of Raney nickel alloy. The "I" and " $H$ " in the image designate the intermediate nickel composition and high the high nickel composition phases, respectively.

resolution achievable at $0.5 \mu$ s peaking time with the SMCD $(\sim 185$ eV FWHM), would be able to handle only about 30,000 cps. Thus, it would require a factor of 10 greater collection time to achieve the same statistics as acquired with the SMCD. This ability to detect and map a minor constituent in a 3-minute acquisition time, using energy dispersive spectrometry, is important for analytical microscopy applications.

In order to test the detector's resistance to electron beam damage, the detector was assembled into a transmission electron microscope (TEM) at the Electron Microscopy Center at Argonne National Lab (ANL). The detector was irradiated continuously with $300 \mathrm{keV}$ backscattered electrons for one hour. Figure 6 shows spectra that were collected before the electron irradiation, and again 10 minutes after the 1 -hour irradiation. No spectral distortion

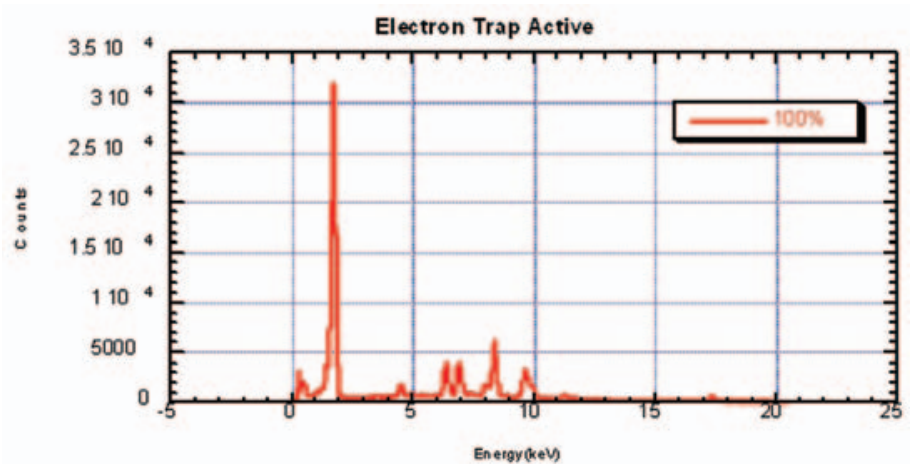

Fig 6a. Spectrum collected before $300 \mathrm{keV}$ electron irradiation.

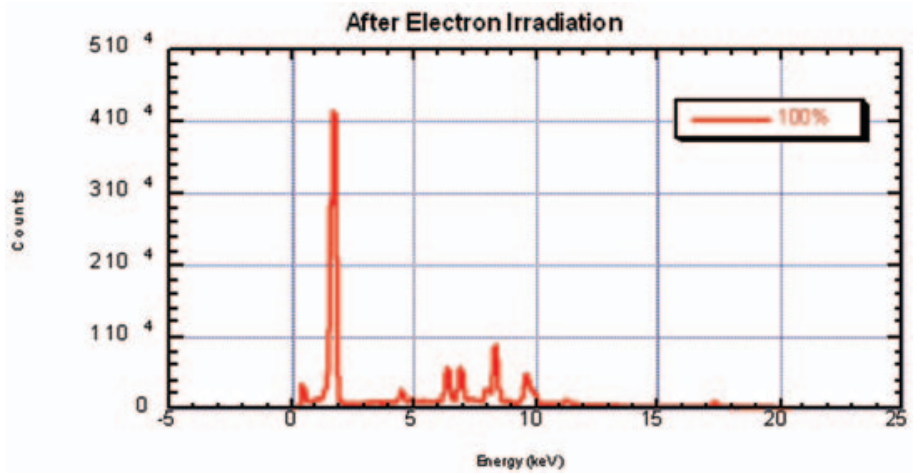

Fig. $6 b$ Spectrum of the same sample as in Fig $6 a$, collected 10 minutes after the 1-hour $300 \mathrm{keV}$ electron irradiation. is observed after irradiation, and the detector returned to its full functionality in less than 10 minutes.

\section{Conclusions:}

The unique features and excellent performance of the SMCD detector make it an ideal candidate for use in many future $\mathrm{X}$ Ray spectroscopy applications. In particular, its extremely high throughput, excellent energy resolution, high stability and near room temperature operation make it very attractive for use in the fields of XRF, XRD and electron beam microanalysis and X-ray mapping. Its "electron hardening" characteristic makes it a stable performance detector at high energy electron environment such as TEM and STEM.

\section{References:}

[1] S. Barkan, et al., "Vortex ${ }^{\circledR}$ - A new high performance silicon drift detector for XRD and XRF Applications", Advances in X-Ray Analysis, Vol. 46 (2003) 332-337.

[2] L. Feng, et al., "A New High Performance Silicon Multi-cathode Detector for $\mathrm{XRD}$ and XRF

Applications", Hard X-ray and Gamma-ray Detector Physics V, Proceedings of SPIE, International Society of Optical Engineering, Vol. 5198, (2004) 103-110.

[3] J. S. Iwanczyk, et al., "Large Area Silicon Drift Detectors for X-Rays- New Results", IEEE Trans. Nucl.

Sci. vol. 46 (1999) 284-288.

[4] U.S Patent \#6,455,858 B1 "Semiconductor Radiation Detector", 2002.

Acknowledgements:

This work was supported in part by NIST and ANL.

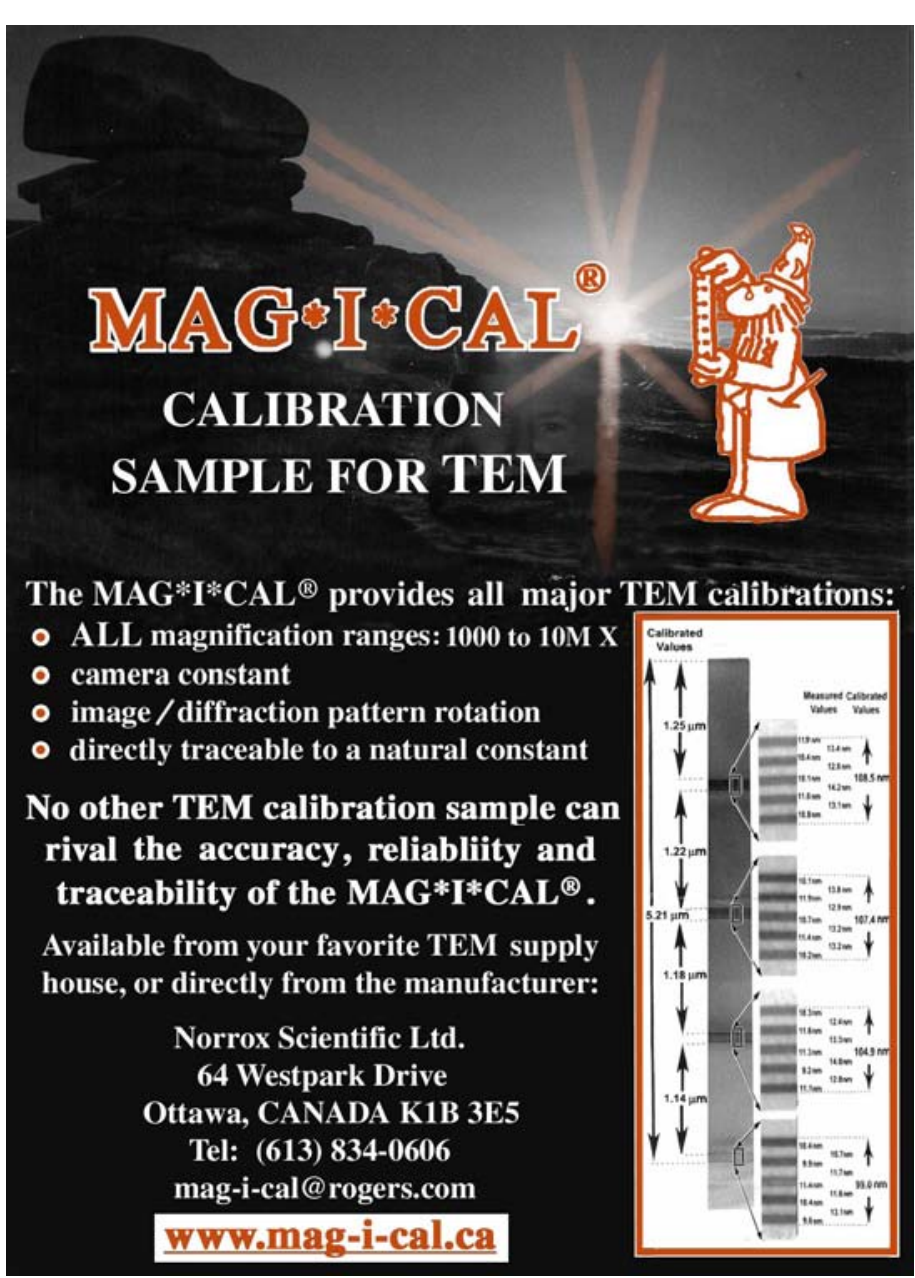

